BNL - 52541

UC - 414

\title{
AN OVERVIEW ON THE LONGITUDINAL STERN-GERLACH EFFECT
}

\author{
M. Conte, W. W. MacKay and R. Parodi
}

November 17, 1997

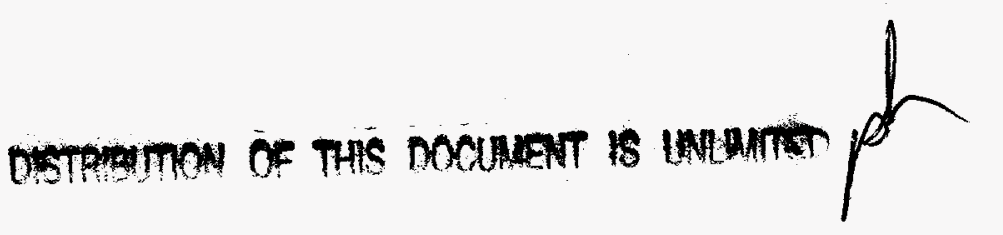

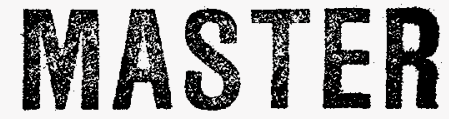

\section{RHIC Project} \\ Brookhaven National Laboratory \\ Associated Universities, Inc. \\ Upton, Long Island, New York 11973
}

'Under Contract No. DE-AC02-76CH00016 with the UNITED STATES DEPARTMENT OF ENERGY 


\section{DISCLAIMER}

This report was prepared as an account of work sponsored by an agency of the United States Government. Neither the United States Government nor any agency thereof, nor any of their employees, not any of their contractors, subcontractors, or their employees, makes any warranty, express or implied, or assumes any legal liability or responsibility for the accuracy, completeness, or usefulness of any information, apparatus, product, or process disclosed, or represents that its use would not infringe privately owned rights. Reference herein to any specific commercial product, process, or service by trade name, trademark, manufacturer, or otherwise, does not necessarily constitute or imply its endorsement, recommendation, or favoring by the United States Government or any agency, contractor, or subcontractor thereof. The views and opinions of authors expressed herein do not necessarily state or reflect those of the United States Government or any agency, contractor or subcontractor thereof.

Printed in the United States of America

Available from

National Technical Information Service

U.S. Department of Commerce 5285 Port Royal Road

Springfield, VA 22171 


\section{DISCLAIMER}

Portions of this document may be illegible electronic image products. Images are produced from the best available original document. 


\title{
An Overview on the Longitudinal Stern-Gerlach Effect
}

\author{
M. Conte ${ }^{1}$, W.W. MacKay ${ }^{2}$ and R. Parodi ${ }^{3}$. \\ (1) Dipartimento di Fisica dell'Università di Genova, INFN Sezione di Genova, \\ Via Dodecaneso 33, 16146 Genova, Italy. \\ (2) RHIC Project, Brookhaven National Laboratory, Upton, NY 11973, USA. \\ (3) INFN Sezione di Genova, Via Dodecaneso 33, 16146 Genova, Italy.
}

\section{Introduction}

The Stern-Gerlach force acts on polarized particles crossing inhomogeneous magnetic fields, $\vec{B}$. In a reference frame where particles are at rest, its expression

$$
\vec{f}_{S G}=-\nabla U
$$

can be deduced from the magnetic potential

$$
U=-\vec{\mu} \cdot \vec{B}
$$

with the magnetic moment

$$
\vec{\mu}=g \frac{e}{2 m} \vec{S}
$$

Here $e= \pm 1.602 \times 10^{-19} \mathrm{C}$ is the elementary charge with + for protons and - for antiprotons, making $\vec{\mu}$ and $\vec{S}$ either parallel or antiparallel, respectively. Besides $m$ is the $p, \bar{p}$ rest mass $\left(1.67 \times 10^{-27} \mathrm{~kg}\right)$ and the relation between the gyromagnetic ratio $g$ and the anomaly $a$ is $a=\frac{g-2}{2}$ with

$$
a=1.793 \quad(g=5.586) \quad \text { for } p, \bar{p}
$$

In the rest system, the quantum vector $\vec{S}$, called spin, has modulus $|\vec{S}|^{2}=s(s+1) \hbar^{2}$ and its component parallel to the magnetic field lines can take the following values only:

$$
S_{m}=(-s,-s+1, \ldots, s+1, s) \hbar
$$

where $\hbar=1.05 \times 10^{-34} \mathrm{Js}$ the reduced Planck's constant. Combining Eqs. (3) and (5) we obtain for a generic spin- $\frac{1}{2}$ fermion

$$
\mu=|\vec{\mu}|=g \frac{|e| \hbar}{4 m}
$$


and for $p, \bar{p}$ in particular

$$
\mu=1.41 \times 10^{-26} \mathrm{JT}^{-1}
$$

The Lorentz transformation of any four-vector $\left(V_{0}, \vec{V}\right)$ from the moving frame, labeled with $R$, where particles are at rest, to the laboratory system, tagged with $L$, is:

$$
\left(\begin{array}{c}
V_{L 0} \\
V_{L x} \\
V_{L y} \\
V_{L z}
\end{array}\right)=\left(\begin{array}{cccc}
\gamma & 0 & \beta \gamma & 0 \\
0 & 1 & 0 & 0 \\
\beta \gamma & 0 & \gamma & 0 \\
0 & 0 & 0 & 1
\end{array}\right)\left(\begin{array}{c}
V_{R 0} \\
V_{R x} \\
V_{R y} \\
V_{R z}
\end{array}\right)=\left(\begin{array}{c}
\gamma\left(V_{R 0}+\beta V_{R y}\right) \\
V_{R x} \\
\gamma\left(\beta V_{R 0}+V_{R y}\right) \\
V_{R z}
\end{array}\right)
$$

having chosen the $y$-axis parallel to the velocity $\vec{v}=\vec{\beta} c$ and where $\beta$ and $\gamma$ are the relativistic parameters of the beam. On the other hand $V_{R y}$ is the projection of $\vec{V}$ on the $y$-axis or, due to our choices, along the velocity direction; consequently we may write:

$$
V_{R y}=\frac{\vec{\beta} \cdot \vec{V}_{R}}{\beta}
$$

Therefore, taking into account both Eqs. (8) and (9), and bearing in mind that $\hat{j}=\frac{\vec{\beta}}{\beta}$, we have:

$$
\vec{V}_{L}=\hat{i} V_{R x}+\hat{j} V_{R y}+\hat{k} V_{R z}+\hat{j}(\gamma-1) V_{R y}+\hat{j} \beta \gamma V_{R 0}
$$

or

$$
\vec{V}_{L}=\vec{V}_{R}+\frac{\gamma-1}{\beta^{2}}\left(\vec{\beta} \cdot \vec{V}_{R}\right) \vec{\beta}+\gamma V_{R 0} \vec{\beta}=\vec{V}_{R}+\frac{\gamma^{2}}{\gamma+1}\left(\vec{\beta} \cdot \vec{V}_{R}\right) \vec{\beta}+\gamma V_{R 0} \vec{\beta}
$$

If we consider as $\vec{V}$, the spin vector $\vec{S}$ in the rest system augmented by a nil fourth component to yield a four-vector ${ }^{1}\left(0, \vec{S}_{R}\right)$, then in a general frame the four-vector has components $\left(\vec{\beta} \cdot \vec{S}_{L}, \vec{S}_{L}\right)$. The transformation for the spin vector is now:

$$
\vec{S}_{L}=\vec{S}_{R}+\frac{\gamma^{2}}{\gamma+1}\left(\vec{\beta} \cdot \vec{S}_{R}\right) \vec{\beta}
$$

\footnotetext{
${ }^{1}$ While this may seem somewhat ad hoc, a more careful treatment[1] by defining an angular momentum tensor as

$$
J^{\alpha \beta}=\int\left(x^{\alpha} T^{\beta 0}-x^{\beta} T^{\alpha 0}\right) d^{3} x,
$$

where $T$ is the stress energy tensor and $x$ is the position four-vector, and we integrate over all of 3-space. This angular momentum contains both the intrinsic spin as well as "orbital" angular momentum. It is possible to remove the orbital momentum by contracting indices of the product of momentum tensor and the particle's four-velocity:

$$
S_{\alpha}=\frac{1}{2} \epsilon_{\alpha \beta \gamma \delta} J^{\beta \gamma} U^{\delta},
$$

where $U=(\gamma, \gamma \vec{\beta})$ and $\epsilon_{\alpha \beta \gamma \delta}$ is the usual four dimensional Levi-Civita tensor.
} 


$$
S_{L 0}=\vec{\beta} \cdot \vec{S}_{L}
$$

having thus obtained the very well known relation [2],[3] which rules the Lorentz transformation of the spin. Similarly we may write

$$
\vec{\mu}_{L}=\vec{\mu}_{R}+\frac{\gamma^{2}}{\gamma+1}\left(\vec{\beta} \cdot \vec{\mu}_{R}\right) \vec{\beta}+\gamma \mu_{R 0} \vec{\beta}
$$

where it will be shown in the next section that the Stern-Gerlach force equation in the lab system is consistent with $\mu_{R 0}=0$.

Under this hypothesis, it is trivial [4],[5],[7] to verify that:

$$
\vec{\mu}_{L}=\vec{\mu}_{R} \quad\left(\text { for } \vec{\beta} \perp \vec{\mu}_{R}\right)
$$

and

$$
\vec{\mu}_{L}=\gamma \vec{\mu}_{R} \quad\left(\text { for } \vec{\beta} \| \vec{\mu}_{R}\right)
$$

Moreover, the Lorentz transformation relating the fields $\vec{B}_{L}, \vec{E}_{L}$, seen in the laboratory frame, to the fields $\vec{B}_{R}, \vec{E}_{R}$, measured in a frame where the particles are at rest, are:

$$
\left\{\begin{array}{l}
\vec{B}_{R \|}=\vec{B}_{L \|} \\
\vec{B}_{R \perp}=\gamma\left(\vec{B}_{L \perp}-\frac{1}{c} \vec{\beta} \times \vec{E}_{L \perp}\right)
\end{array}\right.
$$

and

$$
\left\{\begin{array}{l}
\vec{E}_{R \|}=\vec{E}_{L \|} \\
\vec{E}_{R \perp}=\gamma\left(\vec{E}_{L \perp}+\vec{\beta} c \times \vec{B}_{L \perp}\right)
\end{array}\right.
$$

When $\vec{E}_{L}=0$, Eq. (19) reduces to

$$
\left\{\begin{array}{l}
\vec{B}_{R \|}=\vec{B}_{L \|} \\
\vec{B}_{R \perp}=\gamma \vec{B}_{L \perp}
\end{array}\right.
$$

\section{Stern-Gerlach Force}

In this section we shall evaluate the expression of Stern-Gerlach force in the laboratory frame, where usually we live and operate, in two different ways. One, rather straight forward, consists in applying the magnetic field $\vec{B}_{L}=\vec{B}_{L \perp}+\vec{B}_{L \| \mid}$ to the magnetic moment $\mu_{L}$, as transformed according to Eqs. (17) and (18); for $\vec{B}_{L}$ perpendicular to $\vec{\beta}$ we have:

$$
f_{L \perp}=\left|\vec{f}_{L \perp}\right|=\left|\nabla_{L \perp}\left(\vec{\mu}_{L} \cdot \vec{B}_{L}\right)\right|=\left|\nabla_{L \perp}\left(\vec{\mu}_{R} \cdot \vec{B}_{L \perp}\right)\right|=G_{\perp} \mu_{R}
$$

while for $\vec{B}_{L}$ parallel to $\vec{\beta}$ we have:

$$
f_{L \|}=\left|\vec{f}_{L \|}\right|=\left|\nabla_{L \|}\left(\vec{\mu}_{L} \cdot \vec{B}_{L}\right)\right|=\left|\nabla_{L \|}\left(\gamma \vec{\mu}_{R} \cdot \vec{B}_{L \|}\right)\right|=\gamma G_{\|} \mu_{R}
$$


In the other procedure, the Stern-Gerlach force is evaluated in the moving system and then is transformed into the laboratory frame, by making use of its relativistic extension, the four-force,

$$
F_{\alpha}=\frac{d P_{\alpha}}{d \tau}=\gamma \frac{d P_{\alpha}}{d t} \equiv\left(F_{0}, \vec{F}\right)
$$

where

$$
P_{\alpha} \equiv\left(\frac{E_{\mathrm{tot}}}{c}, \vec{p}\right)
$$

and

$$
E_{\text {tot }}=W_{\text {kin }}+m c^{2}, \quad \vec{p}=\gamma m \vec{v}=\vec{\beta} \gamma m c
$$

Therefore Eq. (24) can be written as

$$
F_{\alpha} \equiv\left(\frac{\gamma}{c} \frac{d E_{\text {tot }}}{d t}, \gamma \vec{f}\right)
$$

or

$$
F_{R, \alpha} \equiv\left(0, \vec{f}_{R}\right)
$$

in the rest frame, where $\gamma_{R}=1, E_{\text {tot }}=m c^{2}=$ constant, and

$$
\vec{f}_{R}=\vec{f}_{R \perp}+\vec{f}_{R \|}=-\nabla_{R \perp} U_{R}-\nabla_{R \|} U_{R}=-\nabla_{L \perp} U_{R}-\gamma \nabla_{L \|} U_{R}
$$

where $\nabla_{L \perp}=\nabla_{R \perp}$, due to the Lorentz invariance of any spatial coordinate perpendicular to the motion direction, and with $\nabla_{L \|}=\gamma \nabla_{R \|}$ since $\frac{\partial}{\partial y_{R}}=\gamma \frac{\partial}{\partial y_{L}}$. Moreover, bearing in mind Eqs. (2) and (21), Eq. (26) may be split as follows:

$$
f_{R \perp}=\left|\vec{f}_{R \perp}\right|=\left|\nabla_{L \perp}\left(\vec{\mu}_{R} \cdot \vec{B}_{R}\right)\right|=\left|\nabla_{L \perp}\left(\vec{\mu}_{R} \cdot \gamma \vec{B}_{R \perp}\right)\right|=\gamma G_{\perp} \mu_{R}
$$

and

$$
f_{R \|}=\left|\vec{f}_{R \|}\right|=\left|\gamma \nabla_{L \|}\left(\vec{\mu}_{R} \cdot \vec{B}_{R \|}\right)\right|=\gamma\left|\nabla_{L \|}\left(\vec{\mu}_{R} \cdot \vec{B}_{L \|}\right)\right|=\gamma G_{\|} \mu_{R}
$$

By applying Eq. (8) to the four-force (25) we obtain:

$$
\left(\begin{array}{c}
F_{L, 0} \\
F_{L, x} \\
F_{L, y} \\
F_{L, z}
\end{array}\right)=\left(\begin{array}{cccc}
\gamma & 0 & \beta \gamma & 0 \\
0 & 1 & 0 & 0 \\
\beta \gamma & 0 & \gamma & 0 \\
0 & 0 & 0 & 1
\end{array}\right)\left(\begin{array}{c}
0 \\
f_{R, x} \\
f_{R, y} \\
f_{R, z}
\end{array}\right)=\left(\begin{array}{c}
\beta \gamma f_{R, y} \\
f_{R, x} \\
\gamma f_{R, y} \\
f_{R, z}
\end{array}\right)
$$

whose space components are respectively for the transverse and longitudinal examples:

$$
\left(\begin{array}{c}
\gamma f_{x} \\
\gamma f_{y} \\
\gamma f_{z}
\end{array}\right)_{L}=\left(\begin{array}{c}
f_{x} \\
0 \\
f_{z}
\end{array}\right)_{R} \text { and }\left(\begin{array}{c}
\gamma f_{x} \\
\gamma f_{y} \\
\gamma f_{z}
\end{array}\right)_{L}=\left(\begin{array}{c}
0 \\
\gamma f_{y} \\
0
\end{array}\right)_{R}
$$

or

$$
f_{L \perp}=G_{\perp} \mu_{R} \text { and } f_{L \|}=\gamma G_{\|} \mu_{R}
$$

having thus found the same expressions as in Eqs. (22) and (23). 


\section{Stern-Gerlach Kicks}

A particle crossing a region, of length $l_{G}$, where the magnetic field gradient acts parallel to the motion direction, experiences a variation $\delta p_{\|}$of its momentum which, in its turns, causes a change, or kick, of the momentum spread

$$
\zeta=\frac{\delta p_{\|}}{p}
$$

Then, bearing in mind the impulse theorem $\delta p_{\|}=\left|\vec{f}_{L \|}\right| \delta t$, with $\vec{f}_{L \|}$ given by Eq. (23) and with $\delta t=\frac{l_{G}}{\beta c}$, Eq. (32) becomes:

$$
\zeta=\frac{\left|\vec{f}_{L\|\|}\right| l_{G}}{\beta^{2} \gamma m c^{2}}= \pm \frac{\gamma G_{\|} \mu_{R} l_{G}}{\beta^{2} \gamma m c^{2}}= \pm \frac{1}{\beta^{2}} \frac{G_{\perp} l_{G}}{B_{\infty}}
$$

where $p=\beta \gamma m c$ and

$$
B_{\infty}=\frac{m c^{2}}{\mu}=\frac{1.503 \times 10^{-10} \mathrm{~J}}{1.41 \times 10^{-26} \mathrm{JT}^{-1}}=10^{16} \mathrm{~T}
$$

for protons. From Eq. (33) we may find

$$
N_{S S}=\frac{\left(\frac{\Delta p}{p}\right)}{\zeta}=\beta^{2} \frac{B_{\infty}}{G_{\|} l_{G}}\left(\frac{\Delta p}{p}\right)
$$

as the number of revolutions required for attaining a momentum separation, between particles with opposite spin states, just equal to $2\left(\frac{\Delta p}{p}\right)$. Note that similar results have already been obtained [8] for a particle crossing a region, of length $l_{G}$, where the magnetic field gradient acts perpendicularly to the motion direction. In this case, the particle experiences a variation $\delta p_{\perp}$ of its transverse momentum which, in its turn, causes a change, or kick, of the trajectory slope

$$
\eta=\frac{\delta p_{\perp}}{p}=\frac{\left|\vec{f}_{L \perp}\right| l_{G}}{\beta^{2} \gamma m c^{2}}=\frac{G_{\perp} \mu_{R} l_{G}}{\beta^{2} \gamma m c^{2}}=\frac{1}{\beta^{2} \gamma} \frac{G_{\perp} l_{G}}{B_{\infty}}
$$

Similarly to what has been done before, we may define as

$$
\left(N_{S S}\right)_{\perp}=\frac{\Delta x^{\prime}}{2 \eta}=\beta^{2} \gamma \frac{B_{\infty}}{2 G_{\perp} l_{G}} \Delta x^{\prime}
$$

where $\Delta x^{\prime}$ is the angular separation between particles with opposite spin states. Both Eqs. (35) and (37) demonstrate that a huge number of revolutions is required in order to have a detectable effect. The transverse kick (36) makes sense at very low energies only, where $\gamma \simeq 1$, and must be carefully synchronized with the betatron oscillations, as extensively discussed in Ref. [8]. Instead at high energy where $\beta \simeq 1$, the longitudinal kick (33) is energy independent. 


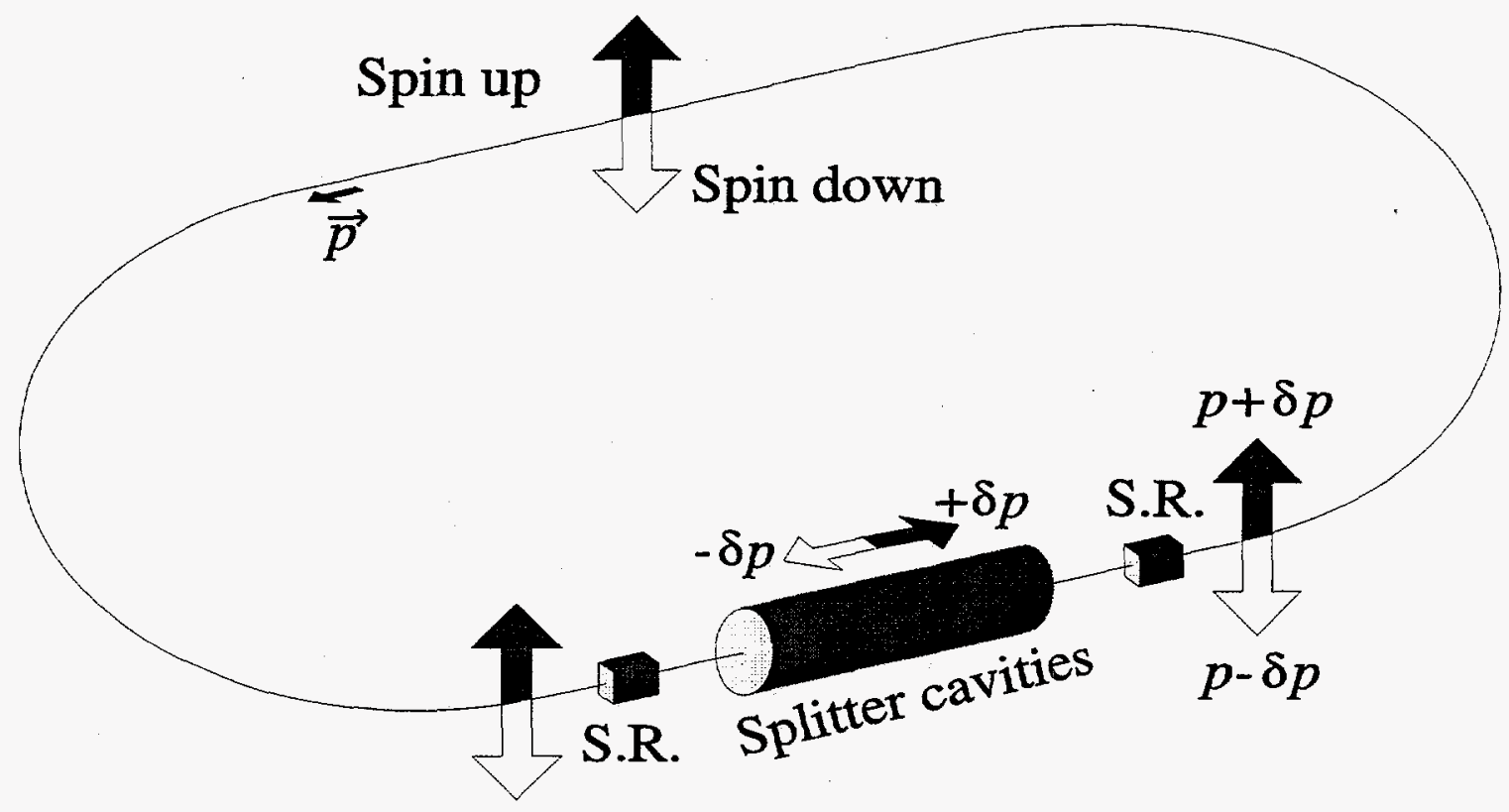

Figure 1: Schematic of model accelerator.

\section{Suggested Procedure}

The total number of protons, $N$, is generally of the order of $10^{11} \rightarrow 10^{13}$. If we define

$N_{\uparrow}=$ No. Particles Spin Up (parallel to $\vec{B}_{\text {ring }}$ )

$N_{\downarrow}=$ No. Particles Spin Down (antiparallel to $\vec{B}_{\text {ring }}$ )

then we may define the polarization of the beam as

$$
P=\frac{N_{\uparrow}-N_{\downarrow}}{N_{\uparrow}+N_{\downarrow}}
$$

where $P$ indicates the macroscopic average over the particle distribution in the beam, which is equivalent to the quantum mechanical expectation value evaluated by the quantum statistical matrix. The polarization $P$ is a quantity varying continuously from 0 to 1 .

An unpolarized beam has $P=0$ or $N_{\uparrow}=N_{\downarrow}$. A spin rotator (see Fig. 1) turns spin-up to e.g. spin-forward and spin-down to spin-backward making Eq. (38) become:

$$
P=\frac{N_{\rightarrow}-N_{\leftarrow}}{N_{\rightarrow}+N_{\leftarrow}}
$$

A suitable (system of) TE rf-cavity accelerates spin-forward particles and deaccelerates spin-backward particles; another spin rotator restores the original situation. The sequences $\left(N_{\uparrow} \rightarrow N_{\rightarrow} \rightarrow N_{\uparrow}\right)$ and $\left(N_{\downarrow} \rightarrow N_{\leftarrow} \rightarrow N_{\downarrow}\right)$ last as long as the beam life-time. Therefore a neat energy separation between particles with opposite spin states will result. 


\section{Longitudinal Magnetic Gradient}

To obtain the wanted Stern-Gerlach effect on the proton beam an intense inhomogeneous magnetic field along the beam direction is required. The use of $\mathrm{rf}$ cavities is an efficient way to build electromagnetic fields. By a careful choice of the operating frequency and the cavity shape a magnetic wave with the right field intensity an shape can be built. It is well known that a particle of unit charge surfing down an rf waveguide increases in energy of an amount equal to the strength of the voltage gradient (the peak electric field of the rf wave moving along the guide synchronous with the particle) times the length of the rf waveguide. In the same way a particle carrying a magnetic moment, moving along a waveguide at the same speed of a magnetic gradient wave, will gain at the end of the travel an energy equal to the waveguide length times the average field gradient times the magnetic moment. In our case we want a strong magnetic field gradient along the beam axis; the natural choice for the rf device is a monopolar $\mathrm{TE}_{01 n}$ cavity having at least one null magnetic field on the cavity axis. By the usual decomposition of the time dependent $\mathrm{rf}$ field inside the cavity into forward and backward traveling waves, the forward traveling wave of magnetic gradient will produce the wanted acceleration (deceleration respectively) of the particle having the spin oriented in the same (opposite) direction as the beam motion. The TE mode is a natural choice due to the strong magnetic field along the cavity axis. By a careful choice of the cavity shape the amplitude of a synchronous magnetic field component (having phase speed equal to the particle speed) can be optimized obtaining quite high field gradients.

As an example the wavelength for a speed-of-light wave at $3 \mathrm{GHz}$ is $0.1 \mathrm{~m}$, a reasonable peak field along the cavity axis is in the range of 0.1-0.2 Tesla, giving an average gradient in the range 40 to 80 Tesla per meter. Our ball-park evaluation of the gradient does not take into account the real shape of the field along the axis. The rough sketch is a triangular wave. That representation is a little pessimistic and some room for improvement is left. Anyway this rough evaluation shows us that a very strong field gradient can be obtained. To have a realistic picture we performed some simulations of TE rf cavities with the Oscar2D [9] code. The provisional result of our work is the cavity shown in Figures 2 and 3. That cavity gives us a longitudinal field gradient of 75 $\mathrm{T} / \mathrm{m}$ at a stored energy of 10 Joules. Surely we have some possibility of improvement, no optimization nor further refinement was attempted; we only rounded the first naive design of a sharp cornered cavity used to guess both frequency and fields. The field distribution of the field gradient along the cavity axis is shown in Fig. 3. The field gradient is big enough to give us the wanted energy gain by using an array of cavities 16 meters long. The rf power dissipation in the cavity wall is quite high, but at the operating frequency of $3 \mathrm{GHz}$ efficient rf generators as klystrons exist and a pulsed operation for the rf system could be foreseen. In that way the overall power consumption can be maintained within reasonable limits. In this logic, a further two way improvement can be proposed. Halving the field gradient and doubling the structure length, the total power consumption is reduced by a factor of two, and the power losses per 
.... LONGITUDINAL MRGNETIC GRADIENT te modes

flle CONTEMO2 frequency $=3013.31 \mathrm{Mhz}$ computed by the OSCAR2D code written by P.FERNANDES and R.PARODI INFN-GENOA

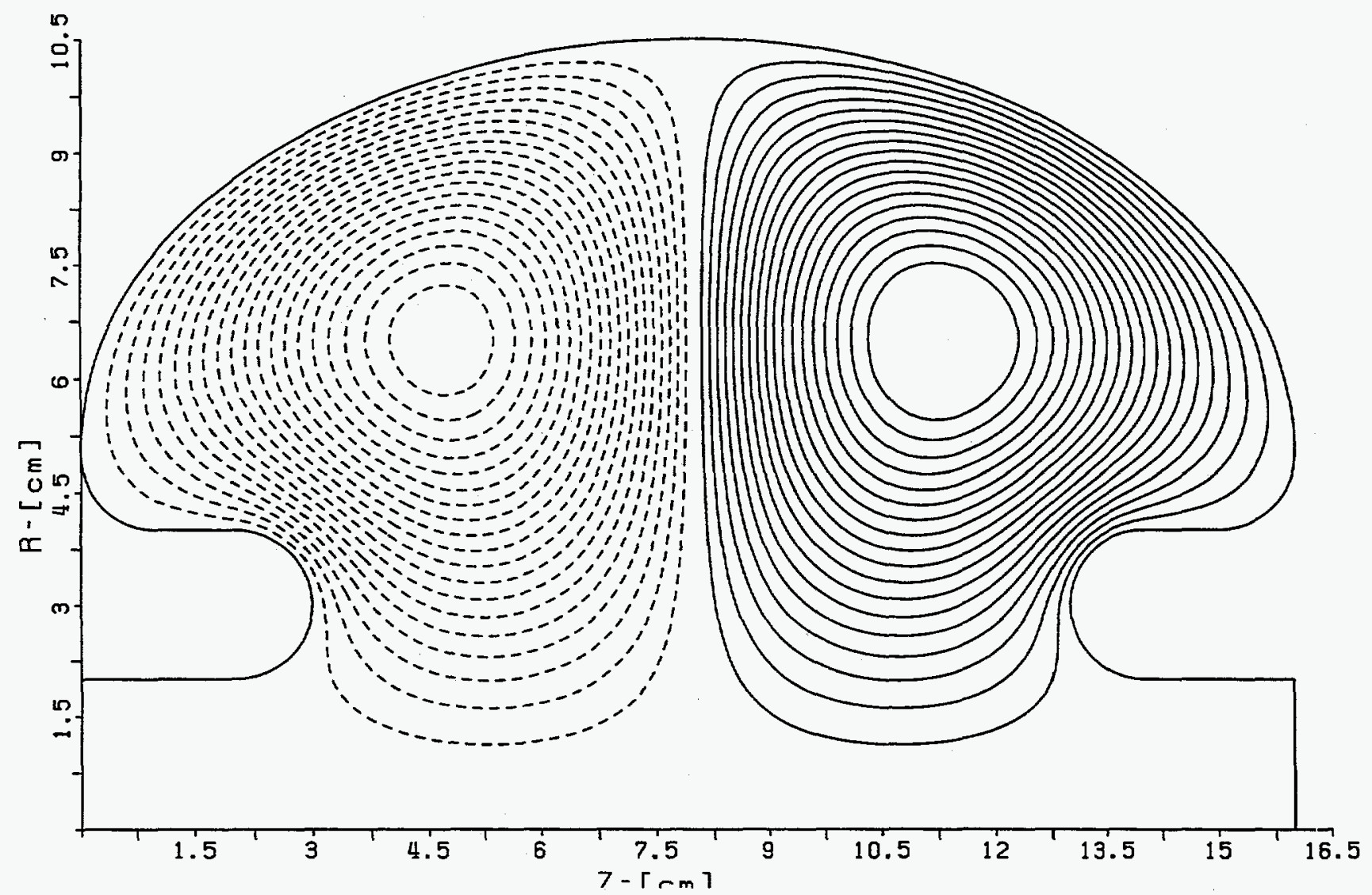

Figure 2: Field plot from OSCAR for the longitudinal magnetic gradient cavity. 


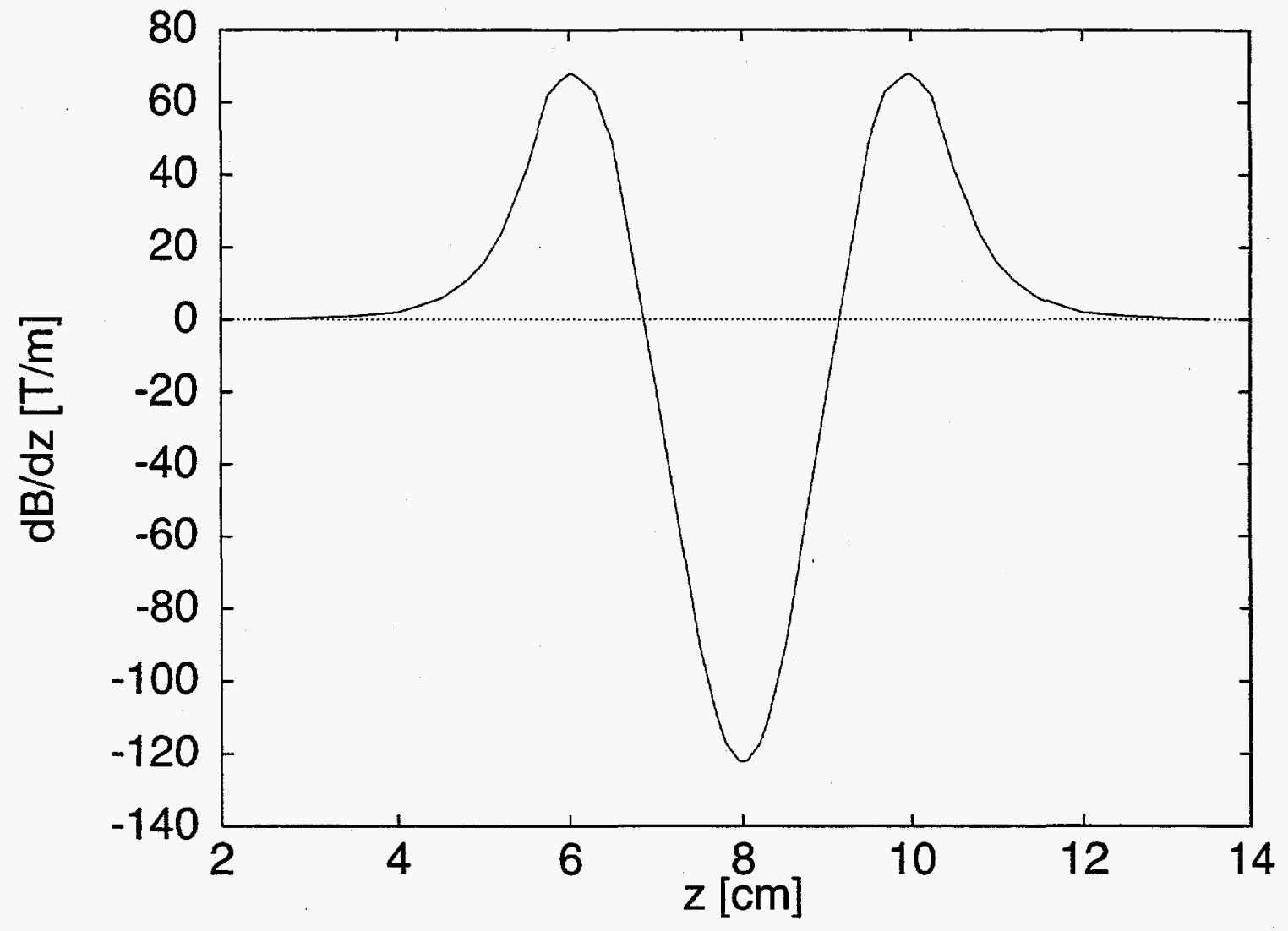

Figure 3: Field along the axis of the longitudinal magnetic gradient cavity. 
cavity reduced by a factor of four. In this way the operating parameter for the spinsplitter structure will fall in the limit of standard rf structures. The last improvement achievable by halving the operating gradient is that the local surface fields in the cavity will fall to $120 \mathrm{mT}$. That value is well below the critical field of the niobium $(200 \mathrm{mT})$ and close to the operating field of $100 \mathrm{mT}$ already obtained for the superconducting cavities of the Tesla Test Facility.

Finally we checked our cavity for unwanted modes close to the one foreseen for spin splitter operation. We computed the first $10 \mathrm{TE}$ and TM modes of the cavity to check for unwanted coalescent bands. The frequency and shunt impedance of the modes are reported in Table I. It is straightforward to see that the frequencies are well separated and we have no harmful degeneration of the modes at the foreseen operation frequency.

Table I: Frequency Modes and Shunt Impedances

\begin{tabular}{|l|l|}
\hline Frequency $(\mathrm{MHz})$ & Shunt Impedance $(\mathrm{Ohm} / \mathrm{m})$ \\
\hline 1026.0 & 1500.00 \\
1415.0 & 365.00 \\
2096.0 & 15.00 \\
2162.0 & \\
2750.0 & 30.00 \\
2833.0 & 44.00 \\
3013.0 & \\
3357.0 & 154.00 \\
3516.0 & \\
3538.0 & 2.00 \\
3900.0 & \\
4248.0 & 0.20 \\
4257.0 & \\
4746.0 & \\
4896.0 & \\
5197.0 & \\
5519.0 & \\
5550.0 & \\
\hline
\end{tabular}

\section{Longitudinal Phase Plane}

We consider a ring with two separate rf systems: a TM cavity for bunching and TE cavities for polarizing. The effect of the TE cavity consists of splitting the original bunch, created by the TM rf bunching system of the ring, into two bunches of particles with opposite spin states, as easily understood by considering the phase oscillation equation for small amplitudes. This is a somewhat oversimplified example, but still valid for giving a general outlook. It is possible to demonstrate that this equation is 


$$
\frac{d^{2} \phi}{d t^{2}}+\Omega_{s}^{2} \phi=\Omega_{s}^{2} k_{\phi} t
$$

with $\Omega_{s}$ being the synchrotron oscillation frequency and

$$
k_{\phi}= \pm \frac{G_{\|} l_{G}}{B_{\infty}} \frac{V_{p}}{V_{\mathrm{RF}}} \frac{\gamma}{\tau}
$$

where $G_{\|} l_{G}$ is the integrated magnetic gradient, $B_{\infty}$ is given by Eq. (34), $V_{p}=938 \mathrm{MeV}$ is the voltage corresponding to the (anti)proton rest mass energy, $V_{\mathrm{RF}}$ is the rf peak voltage and, $\tau$ is the revolution period. The solution of Eq. (40) is:

$$
\phi(t)=\phi_{0} \cos \Omega_{s} t+\frac{\dot{\phi}_{0}-k_{\phi}}{\Omega_{s}} \sin \Omega_{s} t+k_{\phi} t
$$

which clearly demonstrates, according to the sign of $k_{\phi}$, how particles with opposite spin states have two different centers for their phase oscillations drifting slowly apart from each other to form two distinct bunches.

But these two bunches are revolving within the stationary bucket of the TM cavity. Since this rotation would cancel the bunch separation every half synchrotron oscillation period, the longitudinal magnetic gradient $G_{\|}$must be modulated with the same frequency of the phase oscillation, via a time dependent function $F(t)$ which shall be thoroughly discussed later on.

In order to write the synchrotron oscillations equation, we have to consider that the energy gain is either increased (sign + ) or decreased (sign -) by the tiny amount of energy $\gamma \mu G_{\|} l_{G}$, due to the Stern-Gerlach force; then, making use of the canonical variables $(\phi, W)$ in the longitudinal phase plane, we have:

$$
\begin{gathered}
\frac{d \phi}{d t}=C_{\phi} W \\
\frac{d W}{d t}=-C_{\Omega} f(\phi) \mp C_{\mu} F(t)
\end{gathered}
$$

with

$$
\begin{gathered}
C_{\phi}=\frac{\omega_{R F}^{2} \eta_{t r}}{\beta_{s}^{2} \gamma m c^{2}}=\frac{\omega_{s}^{2} h^{2} \eta_{t r}}{\beta_{s}^{2} \gamma m c^{2}} \\
C_{\Omega}=\frac{q V_{R F}}{2 \pi h} \\
C_{\mu}=\frac{\gamma \mu G_{\|} l_{G}}{2 \pi h}
\end{gathered}
$$

Therefore, combining Eqs. (43) and (44) we obtain the following equation:

$$
\frac{d^{2} \phi}{d t^{2}}+\Omega_{s}^{2} f(\phi)=\varepsilon F(t)
$$




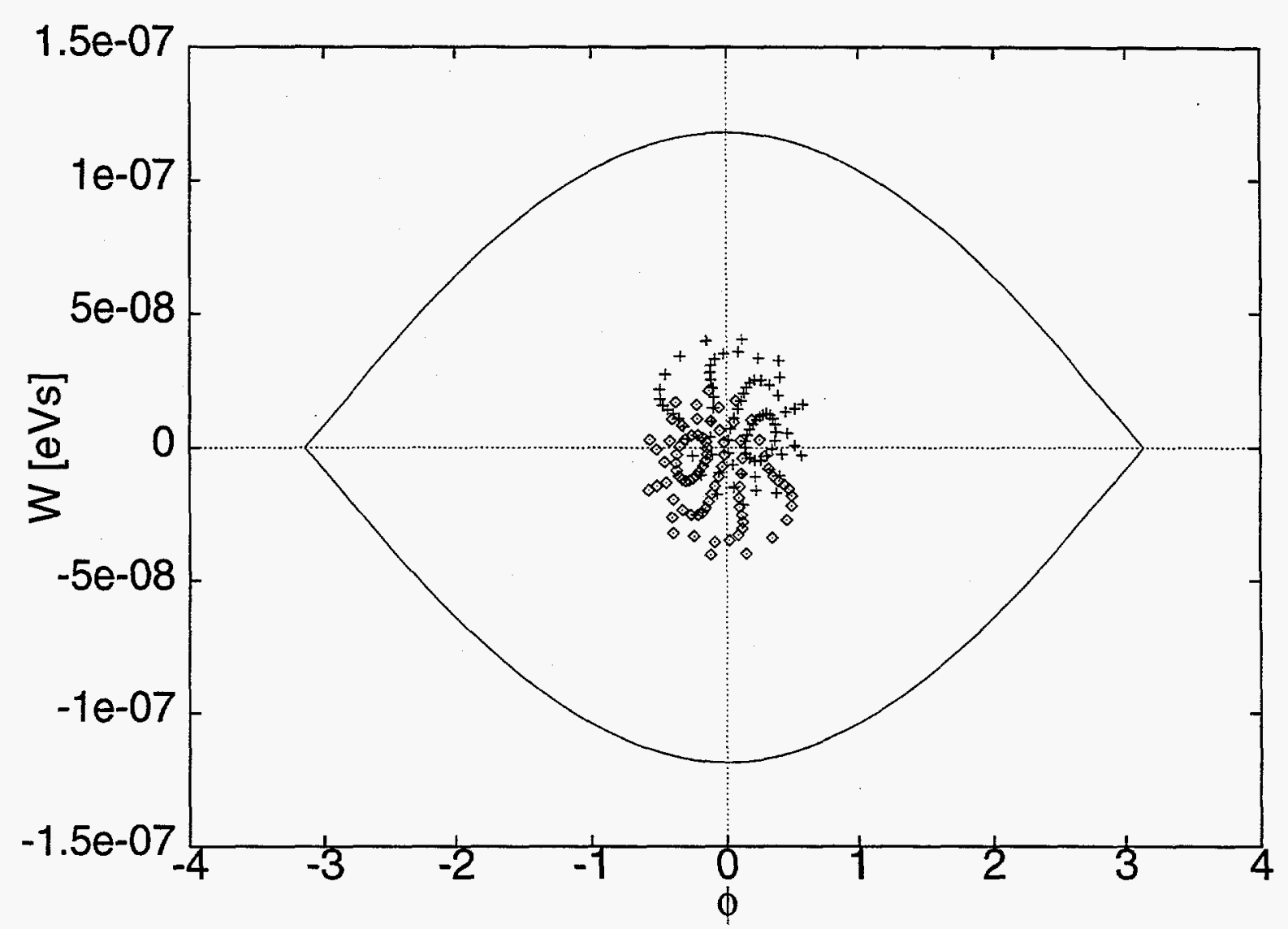

Figure 4: Filamentation of split bunches.

with

$$
\begin{aligned}
\Omega_{s} & =\omega_{s} \sqrt{\frac{h\left|\eta_{t r}\right|}{2 \pi \beta^{2} \gamma} \frac{q V_{R F}}{m c^{2}}} \\
\varepsilon & =\mp \omega_{s}^{2} \frac{h \eta_{t r}}{2 \pi \beta^{2}} \frac{\mu G_{\|} l_{G}}{m c^{2}}
\end{aligned}
$$

and where usually

$$
f(\phi)=\sin \phi \text { and } F(t)=\sin \left(\Omega_{s} t\right)
$$

This sinusoidal law gives rise to filamentation phenomena (see Fig. 4) which destroy the bunch separation. Nevertheless we are studying the possibility of time tagging those particles whose phases are so small that the condition $\sin \phi \simeq \phi$ is fulfilled. In this case, we may also set $F(t)=\sin \left(\Omega_{s} t\right)$ making Eq. (45) reduce to:

$$
\frac{d^{2} \phi}{d t^{2}}+\Omega_{s}^{2} \phi=\varepsilon \sin \left(\Omega_{s} t\right)
$$

which give rise to a phase separation between the two opposite spin states of the following kind: 
Table II: Small Ring for Antiproton

\begin{tabular}{|l|l|}
\hline Relativistic Parameters & $\beta=0.5$ \\
& $\gamma=1.155$ \\
Kinetic Energy & $W=145 \mathrm{MeV}$ \\
Momentum & $p=0.542 \mathrm{GeV} / \mathrm{c}$ \\
Ring Circumference & $L=60 \mathrm{~m}$ \\
Momentum-spread & $\frac{\Delta p}{p}=10^{-5}$ \\
Revolution Period & $\tau=0.4 \mu \mathrm{s}$ \\
Harmonic Number & $h=100$ \\
Particles per bunch & $N_{b}=3 \times 10^{4}$ \\
Radio Frequency & $f_{R F}=250 \mathrm{MHz}$ \\
RF Peak Voltage & $V_{R F}=7.83 \mathrm{~V}$ \\
Transition Energy & $\gamma_{t r}=3$ \\
Transition Parameter & $\eta_{t r}=\gamma^{-2}-\gamma_{\mathrm{tr}}^{-2}=0.639$ \\
\hline
\end{tabular}

$$
\Delta \phi=\frac{\varepsilon}{\Omega_{s}} t \sin \left(\Omega_{s} t\right)
$$

or

$$
(\Delta \phi)_{\mathrm{Max}}=\frac{\varepsilon t}{\Omega_{s}}=\frac{\varepsilon N \tau}{\Omega_{s}}=\frac{2 \pi \varepsilon N}{\omega_{s} \Omega_{s}}
$$

where $N$ is the number of turns and $\tau$ is the revolution period; hence:

$$
(\Delta \phi)_{\mathrm{Max}}=N \frac{\mu G_{\|} l_{G}}{\beta} \sqrt{\frac{2 \pi h \eta_{t r} \gamma}{m c^{2} q V_{R F}}}
$$

Should the request of having polarized antiproton become impelling, an ad hoc small ring could be devised (see Table II), where the saw-tooth wave-form could be implemented with the approximation $f(\phi)=\phi$ for $\phi \in\left(-\frac{\pi}{2}, \frac{\pi}{2}\right)$. Notice that the proposed TE rf cavities dictate $\beta=0.5$ as minimum value. At these very low energies, longitudinal space charge effects can take place and Eq. (45) transforms into:

$$
\frac{d^{2} \phi}{d t^{2}}+\left(\Omega_{s}^{2}-\kappa\right) \phi=\varepsilon \sin \left(\sqrt{\Omega_{s}^{2}-\kappa}\right) t
$$

with $\Omega_{s}$ and $\varepsilon$ as before and

$$
\kappa=\frac{12 g N_{b} \eta_{t r} r_{0} c^{2}}{l_{b}^{3} \gamma^{3}}
$$

where

$$
r_{0}=\frac{q^{2}}{4 \pi \varepsilon_{0} m c^{2}}
$$

is the classical radius,

$$
l_{b}=B_{f} \frac{L}{h}=\frac{B_{f} \beta c \tau}{h}
$$


is the bunch length for bunching factor $B_{f}$, and

$$
g=1+2 \ln \frac{r_{\text {pipe }}}{r_{\text {beam }}}
$$

is a geometric factor taking into account the ratio of beam pipe radius $r_{\text {pipe }}$ to beam radius $r_{\text {beam. }}$. Eq. (51) can be written as

$$
\kappa=\frac{3 g N_{b} \eta_{t r} h^{3} q^{2}}{\pi \varepsilon_{0} B_{f}^{3} L^{3} \gamma^{3} m}
$$

while the modified frequency of Eq. (50) is

$$
\Omega_{s}^{2}-\kappa=\omega_{s}^{2} \frac{h\left|\eta_{t r}\right|}{2 \pi \beta^{2} \gamma} \frac{q}{m c^{2}}\left(V_{R F}-V_{s . c .}\right)
$$

with the effective space charge voltage

$$
V_{\text {s.c. }}=6\left(\frac{\beta c}{\omega_{s} L}\right)^{2} g \frac{N_{b} h^{2} q}{\gamma^{2} B_{f}^{3} \varepsilon_{0} L}=\frac{3}{2 \pi^{2}} g \frac{N_{b} h^{2} q}{\gamma^{2} B_{f}^{3} \varepsilon_{0} L} \simeq 6.83 \mathrm{~V}
$$

where

$$
\frac{\beta c}{\omega_{s} L}=\frac{\beta c \tau}{2 \pi L}=\frac{1}{2 \pi}
$$

Hence Eq. (49) transforms into:

$$
(\Delta \phi)_{M a x}=N \frac{\mu G_{\|} l_{G}}{\beta} \sqrt{\frac{2 \pi h \eta_{t r} \gamma}{m c^{2} q\left(V_{R F}-V \text { s.c. }\right)}}
$$

Still with an integrated gradient $G_{\|} l_{G}=10^{3} \mathrm{~T}$, Eq. (52) would yield a separation of $1 \mathrm{rad} \simeq 57^{0}$ after $8.2 \times 10^{6}$ revolutions.

\section{$7 \quad$ Basic Physical Considerations}

A magnetic field, independent of $\theta$ and with a constant longitudinal gradient $G_{\|}$, fulfills the Maxwell equation for Gauss' law of magnetic fields written in cylindrical coordinates

$$
\nabla \cdot \vec{B}=\frac{1}{r} \frac{\partial}{\partial r}\left(r B_{r}\right)+G_{\|}=0
$$

or

$$
\frac{d}{d r}\left(r B_{r}\right)=-r G_{\|}
$$

from which we obtain:

$$
B_{r}=-\frac{1}{r} \int_{0}^{r} r^{\prime} G_{\|} d r^{\prime}=-\frac{1}{2} G_{\|} r
$$


The total field may then be written as

$$
\vec{B} \equiv\left\{\begin{array}{l}
B_{r}=-\frac{1}{2} G_{\|} r \\
B_{\theta}=0 \\
B_{z}=G_{\|} z
\end{array}\right.
$$

which can be described by the simplest vector potential

$$
\vec{A} \equiv\left\{\begin{array}{l}
A_{r}=0 \\
A_{\theta}=\frac{1}{2} G_{\|} r z \\
A_{z}=0
\end{array}\right.
$$

as can be verified through the equation

$$
\vec{B}=\nabla \times \vec{A}
$$

whose right hand side

$$
\left\{\begin{array}{l}
(\nabla \times \vec{A})_{r}=\frac{1}{r} \frac{\partial A_{z}}{\partial \theta}-\frac{\partial A_{\theta}}{\partial z} \\
(\nabla \times \vec{A})_{\theta}=\frac{\partial A_{r}}{\partial z}-\frac{\partial A_{z}}{\partial r} \\
(\nabla \times \vec{A})_{z}=\frac{1}{r} \frac{\partial}{\partial r}\left(r A_{\theta}\right)-\frac{1}{r} \frac{\partial A_{r}}{\partial \theta}
\end{array}\right.
$$

yields

$$
\left\{\begin{array}{l}
(\nabla \times \vec{A})_{r}=\frac{1}{r} \frac{\partial}{\partial \theta}(0)-\frac{\partial}{\partial z}\left(\frac{1}{2} G_{\|} r z\right)=-\frac{1}{2} G_{\|} r \\
(\nabla \times \vec{A})_{\theta}=\frac{\partial}{\partial z}(0)-\frac{\partial}{\partial r}(0)=0 \\
(\nabla \times \vec{A})_{z}=\frac{1}{r} \frac{\partial}{\partial r}\left(\frac{1}{2} G_{\|} z r^{2}\right)-\frac{1}{r} \frac{\partial}{\partial \theta}(0)=G_{\|} z
\end{array}\right.
$$

coinciding with Eq.(56).

Changing to rectangular coordinates, it easy to demonstrate that a vector of the type $\vec{A} \equiv\left(0, A_{\theta}, 0\right)$ will have the following components:

$$
\vec{A} \equiv\left\{\begin{array}{l}
A_{x}=-A_{\theta} \sin \theta \\
A_{y}=A_{\theta} \cos \theta \\
A_{z}=0
\end{array}\right.
$$

with

$$
\left\{\begin{array}{l}
\sin \theta=\frac{y}{r} \\
\cos \theta=\frac{x}{r} \\
r=\sqrt{x^{2}+y^{2}}
\end{array}\right.
$$

Bearing in mind Eq. (57), Eq. (61) transforms into:

$$
\left\{\begin{array}{l}
A_{x}=-\frac{1}{2} G_{\|} y z \\
A_{y}=\frac{1}{2} G_{\|} x z \\
A_{z}=0
\end{array}\right.
$$

which, due to Eq. (58), gives as components of the magnetic field:

$$
\vec{B}=\nabla \times A=\left(\begin{array}{ccc}
\hat{i} & \hat{j} & \hat{k} \\
\frac{\partial}{\partial x} & \frac{\partial}{\partial y} & \frac{\partial}{\partial z} \\
-\frac{1}{2} G_{\|} y z & \frac{1}{2} G_{\|} x z & 0
\end{array}\right) \equiv\left\{\begin{array}{l}
-\frac{1}{2} G_{\|} x=G_{H} x \\
-\frac{1}{2} G_{\|} y=G_{V} y \\
G_{\|} z
\end{array}\right.
$$


Taking into account Eq. (64) and the magnetic Gauss' law in Cartesian coordinates this time, we have:

$$
\nabla \cdot \vec{B}=-\frac{1}{2} G_{\|}-\frac{1}{2} G_{\|}+G_{\|}=G_{H}+G_{V}+G_{\|}=0
$$

which shows that both horizontal and vertical betatron oscillations are affected by repetitive Stern-Gerlach kicks. Nevertheless, these kicks do not add up since their actions change direction every half revolution of the representative points in both phase planes, unless one of those quite sophisticated solutions thoroughly discussed in Ref. $[8]$ is implemented.

\section{The Unlikelihood of Sitting on Transition}

Another hair-brained idea might be the one of operating just at the ring's transition energy, since in this case synchrotron oscillations should not take place thus eliminating those unwanted effects such cancellation of the kicks adding up and everything else related to the longitudinal phase plane phenomena. By definition we have as transition parameter

$$
\eta_{\mathrm{tr}}=\frac{1}{\gamma^{2}}-\frac{1}{\gamma_{\mathrm{tr}}^{2}}=\frac{\gamma_{\mathrm{tr}}^{2}-\gamma^{2}}{\left(\gamma \gamma_{\mathrm{tr}}\right)^{2}}
$$

Bearing in mind the trivial fact that an energy spread does exist, that is:

$$
\gamma=\gamma_{\mathrm{tr}} \pm \Delta \gamma
$$

Eq. (66) gives:

$$
\eta_{\mathrm{tr}} \simeq \mp \frac{2 \Delta \gamma}{\gamma_{\mathrm{tr}}^{3}}=\mp \frac{2 \beta_{\mathrm{tr}}^{2}}{\gamma_{\mathrm{tr}}^{2}} \frac{\Delta p}{p} \simeq \mp \frac{2}{\gamma_{\mathrm{tr}}^{2}} \frac{\Delta p}{p}
$$

therefore the well known [10] formula

$$
\frac{\Delta \tau}{\tau}=-\eta_{\mathrm{tr}} \frac{\Delta p}{p}
$$

yields, if combined with Eq. (68), the following relation:

$$
\frac{\Delta \tau}{\tau} \simeq \pm \frac{2}{\gamma_{\text {tr }}^{2}}\left(\frac{\Delta p}{p}\right)^{2}
$$

Moreover, the debunching time [10] becomes now:

$$
\tau_{d} \geq \frac{\tau}{4 h\left(\frac{\Delta p}{p}\right)^{2}} \gamma_{\mathrm{tr}}^{2}
$$


The number of revolutions required for attaining the bunch merging is then

$$
N_{d}=\frac{\tau_{d}}{\tau} \geq \frac{\gamma_{\mathrm{tr}}^{2}}{4 h\left(\frac{\Delta p}{p}\right)^{2}}
$$

On the other hand, one usually tries to avoid transition because of the destructive effects [11][12][13] related to the microwave beam-environment. Two quantities describe mainly this effect: the longitudinal stability criterion:

$$
\left|\frac{Z_{\|}}{n}\right| \leq \frac{1}{2} F\left|\eta_{\mathrm{tr}}\right| \beta^{2} \gamma \frac{V_{p}}{\hat{I}}\left(\frac{\Delta p}{p}\right)^{2}
$$

and the exponential growth time characterizing such an instability;

$$
\tau_{\mu}=\tau \sqrt{\frac{\beta^{2} \gamma\left(V_{p} / \hat{I}\right)}{2 \pi\left|\eta_{\mathrm{tr}}\right| n^{2}\left|Z_{\|} / n\right|}}
$$

where

$Z_{\|}=$longitudinal impedance;

$n=$ microwave mode number;

$F=$ form factor (often set equal to 0.65 for proton bunches);

$V_{p}=938 \mathrm{MV}$

$\hat{I}=$ peak current $=($ particles per bunch $) /($ bunch duration $)$;

$\frac{\Delta p}{p}=$ r.m.s. momentum spread.

At a first glance it is clear that, for $\eta_{\text {tr }}$ approaching zero, the stability criterion (73) can hardly be fulfilled but, fortunately, the instability growth time (74) becomes very big, even though the bad effect $\left(\propto\left|\eta_{\mathrm{tr}}\right|\right)$ acts faster than the good one $\left(\propto\left|\eta_{\mathrm{tr}}\right|^{-\frac{1}{2}}\right)$. However, by substituting Eq. (68) into both Eqs. (73) and (74), we obtain:

$$
\left|\frac{Z_{\|}}{n}\right| \leq F \frac{\beta_{\mathrm{tr}}^{4}}{\gamma_{\mathrm{tr}}} \frac{V_{p}}{\hat{I}}\left|\frac{\Delta p}{p}\right|^{3} \simeq \frac{F}{\gamma_{\mathrm{tr}}} \frac{V_{p}}{\hat{I}}\left|\frac{\Delta p}{p}\right|^{3}
$$

and

$$
n N_{\mu}=n \frac{\tau_{\mu}}{\tau}=\frac{\gamma_{\mathrm{tr}}^{2}}{2 \sqrt{\pi}\left(\frac{\Delta p}{p}\right)^{2}}
$$

At this stage we may work out a few numerical values, e.g. by considering operation of RHIC [14] at transition with protons. The essential machine parameters and the quantities characterizing the microwave interaction are gathered in Table III. The debunching takes place well before the wanted energy splitting; nevertheless, the required condition $N_{S S}<N_{d}$ gives:

$$
\left(\frac{\Delta p}{p}\right)<\left[\frac{G_{\|} l_{G}}{B_{\infty}} \frac{\gamma_{\mathrm{tr}}}{4 h}\right]^{\frac{1}{3}} \simeq 1.2 \times 10^{-5}
$$


Table III: RHIC (proton mode)

\begin{tabular}{|l|l|}
\hline$\gamma_{\text {tr }}$ & 22.8 \\
$F$ & 0.65 \\
$\frac{\Delta p}{p}$ & $4.1 \times 10^{-3}$ \\
$I$ & $0.13 \mathrm{~A}$ \\
$h$ & 360 \\
$\left|\frac{Z_{\| 1}}{n}\right|$ & $\leq 14.2 \Omega$ \\
$n N_{\mu}$ & $8.76 \times 10^{6}$ turns \\
& $(110 \mathrm{~s})$ \\
$N_{d}$ & $2.15 \times 10^{4}$ turns \\
& $(0.3 \mathrm{~ms})$ \\
$N_{S S}$ & $4.1 \times 10^{10}$ turns \\
& $(146 \mathrm{~h})$ \\
\hline
\end{tabular}

But, if we use this value in Eq. (75), the impedance limit becomes ridiculously small:

$$
\left|\frac{Z_{\|}}{n}\right|=\frac{F}{4 h} \frac{G_{\|} l_{G}}{B_{\infty}} \frac{V_{p}}{\hat{I}} \simeq 3 \times 10^{-7} \Omega
$$

\section{Objections and Counter-Objections}

A typical objection is that the Stern-Gerlach impulses are too small compared to the impulses arising from spurious forces of any kind. But, while the latter are at random, the former must be coherent, otherwise no separation can take place. This means that, after $N_{\text {turn }}$ revolution, the small Stern-Gerlach signals adding up to the effective strength

$$
V_{S S}=N_{\text {turn }} \bar{v}_{\text {kick }}
$$

while for the random signal we have:

$$
V_{n}=\frac{\bar{v}_{\text {random }}}{\sqrt{N_{\text {turn }}}}
$$

Therefore, combining Eqs. (79) and (80) we obtain as signal to noise ratio:

$$
r_{n}=\frac{V_{S S}}{V_{n}}=N_{\text {turn }}^{\frac{3}{2}}\left(\frac{\bar{v}_{\text {kick }}}{\bar{v}_{\text {random }}}\right)
$$

For $N_{\text {turn }}=10^{6}$, a reasonable number of turns, Eq. (81) yields $r_{n}=1$, even for

$$
\frac{\bar{v}_{\text {kick }}}{\bar{v}_{\text {random }}}=10^{-9}
$$


If we consider unwanted TM modes in the splitter cavities with an unreasonably large longitudinal electric field of $E_{\|}=10^{4} \mathrm{Vm}^{-1}$, the magnitude of the electric and magnetic forces would be of the order of

$$
F_{E}=q E_{\|} \sim\left[10^{-19} \mathrm{C}\right] \times\left[10^{4} V m^{-1}\right]=10^{-15} \mathrm{~N}
$$

and

$$
F_{M}=\mu G_{\|} \sim\left[10^{-24} \mathrm{JT}^{-1}\right] \times\left[10^{2} \mathrm{Tm}^{-1}\right]=10^{-24} \mathrm{~N}
$$

Combining the Eqs. (83) and (84), we obtain:

$$
\frac{F_{M}}{F_{E}} \sim 10^{-9}
$$

i. e., the same tolerable value as in Eq. (82). At this stage, one could comment that such arguments have not stopped all the projects dealing with gravitational wave antennas, where the most favorable sensitivities are of the order of $10^{-12} \ldots$

Greater attention should be paid about matching [15] the external rf modulation of the splitter cavity amplitude to the synchrotron oscillations. This case is different than the one [16] of transverse Stern-Gerlach kicks, where both oscillator and forcing system inherently coincide with the ring lattice. The condition to be fulfilled is that

$$
\delta \Omega_{s} t \ll 2
$$

where $\delta \Omega_{s}$ is the permitted error in matching the modulator frequency to the synchrotron frequency $\Omega_{s}$, and $t$ is the time duration along which the forcing term acts over the oscillator. We then have:

$$
t \ll \frac{2}{\Omega_{s}} \frac{1}{\frac{\delta \Omega_{s}}{\Omega_{s}}}
$$

Choosing RHIC as an example we have $\Omega_{s} / 2 \pi \leq 333 \mathrm{~Hz}$. Since it is possible to obtain [17] a precision $\frac{\delta \Omega_{s}}{\Omega_{s}}$ of the order of $10^{-9}$, using divided-quartz and thermal stabilized oscillators, then Eq. (87) gives:

$$
t \ll 9.56 \times 10^{5} \mathrm{~s} \simeq 266 \mathrm{~h}
$$

well beyond the length of a typical fill ( $\leq 10 \mathrm{~h}$ ).

\section{Conclusions}

The TE rf cavity system is undoubtedly very appealing since it gives the possibility of polarizing both proton and antiproton beams at the end of their acceleration cycles. Practically, this process is similar to the $e^{ \pm}$self-polarization induced by synchrotron radiation. Should this method prove unsuccessful, the small polarizer ring can still be used for attaining polarized antiprotons. In fact, the rf requirements for the low 
energy ring are less demanding than for high energy colliders: a saw-tooth wave-form can be implemented, and the spin separation can be undergone by successive bursts of quasi-monoenergetic antiprotons.

Stochastic cooling techniques, such as momentum to frequency pick-ups, can be directly useful for both methods, either in the very first tests or in the final operating modes. These techniques could also be employed in order to carry out experiments of elementary particles physics, even in presence of filamentation phenomena. In fact, the two bunches of particles with opposite spin states have a core whose particles will always undergo synchrotron oscillations with small amplitude, i.e. will never be entangled by filamentation. Therefore, these innermost particles could be time-tagged and used in either collider or fixed target experiments. Besides, when the bunch cores become empty, a rf reshuffling could pour other particles into them and this process can be iterated. 


\section{References}

[1] S. Weinberg, Gravitation and Cosmology: Principles and Applications of the General Theory of Relativity, John Wiley and Sons, 1972.

[2] K. Hagedorn, Relativistic Kinematics, W.A. Benjamin Inc., 1963.

[3] J.D. Jackson, Classical Electrodynamics, John Wiley and Sons, 1975.

[4] M. Conte, A. Penzo and M. Pusterla, Il Nuovo Cimento, A108 (1995) 127.

[5] N. Akchurin et al,, Evolution of The Spin-Splitter Concept, Proceedings of the Adriatico Research Conference on "Trends in Collider Spin Physics", 5-8 December 1995, Trieste, Eds. Y. Onel, N. Paver and A. Penzo, World Scientific, p. 202 (1997),

[6] N. Akchurin et al., Spin States Separation Based on the Longitudinal Stern-Gerlach Effect, Proceedings of the SPIN96 Symposium, 10-14 September 1996, Amsterdam, Eds. C.W. de Jager, T.J. Ketel, P.J. Mulders, J.E.J. Oberski and M. OskamTamboezer, p. 263., World Scientific, Singapore (1997),

[7] M.Conte, R. Jagannathan, S.A. Khan and M. Pusterla, Particle Accelerators, 56 (1996) 99.

[8] M. Conte, A. Penzo, A. Pisent and M. Pusterla, "Analytical Treatment of the Spin-Splitter", Internal Report INFN/TC-88/25.

[9] P. Fernandes and R. Parodi, "Oscar2D User's Guide", INFN/TC-90/04.

[10] M. Conte and W.W. MacKay, An Introduction to the Physics of Particle Accelerators, World Scientific (1991).

[11] A.G. Ruggiero and V.G. Vaccaro, "Solution of the dispersion relation for longitudinal resistive stability of an intense coasting beam in a circular accelerator" (application to the ISR), CERN-ISR-TH/68-33 (1968).

[12] E. Keil and W. Schnell, "Concerning longitudinal stability in the ISR", CERNISR-TH-RF/69-48 (1969).

[13] A. Hoffman, "Single-Beam Collective Phenomena - Longitudinal", p. 139, CERN 77-13, (1977).

[14] M. A. Harrison, "The RHIC Project", Proceedings of EPAC96, p. 13, Sitges (Barcelona), 1996.

[15] H.G. Hereward, "The elementary theory of Landau damping", CERN 65-20.

[16] M. Conte, Y. Onel, A. Penzo, A. Pisent, M. Pusterla and R. Rossmanith, "The Spin-Splitter Concept", Internal Report INFN/TC-93/04.

[17] R. Parodi, Thesis, University of Genoa. 